posterior hypothalamic activity and 2. release of an oxytrocin-like peptide or its precursor". In all this he does not underrate the possible importance of aldosterone in the long term regulation of extracellular fluid volume.

Rather less satisfactory is the treatment of acid base balance and osmoregulation, mainly because these sections are so brief, but also because some of the statements made are open to question. It is not accurate to say that the anti-diuretic hormone osmoreceptors normally respond to rate of change of osmolarity rather than the absolute level: Verney showed twenty or more years ago that these receptors do not adapt when exposed to sustained small increases in osmotic pressure. Osmoreceptors may give transient responses, but these would not be particularly helpful in the long term regulation of osmotic pressure where some sort of proportional control is required.

Cort is deliberately polemical, for he believes that an unopinionated book seldom makes good reading, and I think many would agree with him. Running through the text is the argument that in classical physiology we usually study an isolated mechanism under rigidly defined conditions and often under anaesthesia. We quite naturally interpret the behaviour of the intact conscious animal in terms of this mechanism, often without taking into account the possibility that there are other unknown and porhaps more important mechanisms which may supplant and certainly modify the original mechanism. Several completely different types of mechanism may function in parallel towards the control of some important variable the stability of which is essential for life. As examples of this we may note that the adrenalectomized rat will maintain reasonable health and sodium balance if provided with saline to drink, and that the serum osmotic pressure in diabetes insipidus is close to normal despite the greatly increased turnover of water. Even in experiments in which we are apparently at pains to study the responses of an animal with all its mochanisms functioning, as in balance studies, we may be deluding ourselves because the animal is usually placed on a constant intake while the renal regulation of some variable such as osmolarity or volume is studied. We must remember that the central nervous system plays a part in both sides of the balance equation, that there is often a change in voluntary intake to a given stress as well as a renal response and that intake and excretion normally interact and modify each other. Cort asks the somewhat enigmatic question ". . . is the whole, working, biological system a sum of its parts, i.e. mechanisms, or does it represent a qualitative quantum jump over the individual regulating mechanisms?"

In short, this is a most stimulating book that covers every aspect of the control of body fluids. It is easy to disagree with many of the ideas that are advanced, but they are put forward with the intention of arousing interest, which they do, and of provoking research, which they will.

J. T. FItZsImons

\section{BETA DECAY IN REVIEW}

\section{The Theory of Beta Radioactivity}

By E. J. Konopinski. (The International Series of Monographs on Physics.) Pp. $\mathrm{x}+403$. (Oxford: Clarendon Press; London: Oxford University Press, 1966.) 75s. net.

\section{Beta Decay}

By C. S. Wu and S. A. Moszkowski. (Interscience Monographs and Texts in Physics and Astronomy, Vol. 16.) Pp. xiv +394. (New York and London: Interscience Publishers, a Division of John Wiley and Sons, 1966.) $120 s$.

THE story of the development of the theory of $\beta$ decay and from this of the theory of weak interactions in general is one of the most fascinating in physics. It began with a puzzle, the continuous $\beta$ spectrum, solved by Pauli by the introduction of the neutrino in 1931. Then, after a period of gradual progress initiated by Fermi in 1933, came that extraordinary volte face in 1956, the breakdown of parity conservation. This was first proposed by Lee and Yang and subsequently verified experimentally by Miss $W u$ and her collaborators in 1957. At once, through the two component theory of the neutrino, came major advances in understanding of $\beta, \pi$ and $\mu$ decay and $a$ satisfactory formulation of low energy weak interaction processes.

A detailed account of this stage in the development is now the subject of a book by one of the early pioneers of the theory of $\beta$ decay-Professor Konopinski. This spells out step by step each stage in the rather complicated calculations including all effects which might be expected to give a measurable contribution. The basic assumptions of the theory are compared with experiment wherever possible and the reader is provided with all those tools of the weak interaction trade appropriate to the low energy domain (although $\beta$-circularly polarized $\gamma$ correlation theory is omitted).

More recently the interrelation of weak interaction theory with other branches of particle physics has led to many important developments. The present fashion for current-current commutation relations has its roots in the work of Goldberger and Trieman in connexion with $\pi$ meson decay, and the ideas of unitary symmetry have, through the Cabibbo angle, explained why even small differences in coupling constants which occur in the theory of transitions conserving strangeness are nevertheless significant. Perhaps most important of all has been the suggestion by analogy with electrodynamics that the weak interactions are mediated by an intermediate vector boson. This, together with the availability of high energy neutrinos from the big accelerators and in the cosmic ray flux, has led to what is now another branch of high energy physies-neutrino physies.

The volume by $\mathrm{Wu}$ and Moszkowski is a review of the subject to this point. With the advantage of a much broader canvas, they have presented an extremely readable account of both the theory and the more recent experiments. The formal details required for a reasonable understanding of the processes by which the relevant numbers are arrived at are reserved for appendixes, a historical introduction is provided and so also is a final chapter on recent developments written, as the authors put it, "with great emotion and enthusiasm". The book is completed by an excellent bibliography.

It is only to be expected, in this field in particular, that nature should have the last word. In 1964, experiments on the decay of neutral $K$ mesons by Christensen and collaborators demonstrated unexpectedly the apparent failure of time reversal invariance, a rather curious echo of the " $\tau-\theta$ puzzle", presented by the decay of the charged $K$ mesons, which led to the developments associated with the breakdown of parity conservation. If certain recent conjectures by Lee are verified, and the experimental evidence is at present conflicting, our understanding of the basic invariance properties of the interactions of elementary particles must shortly undergo another major upheaval.

G. N. FowLER

\section{PUTTING TO SLEEP}

\section{Animal Anaesthesia}

By Melchior Westhues and Rudolf Fritsch. Vol, 2: General Anaesthesia. Translated by A. David Weaver. Pp. xxi +458 . (Edinburgh and London: Oliver and Boyd, Ltd., 1965.) 95s.

THIs is the second of two volumes entitled Die Narkose der Tiere. The first volume is concerned with local 